Received 01/08/2018

Review began 01/25/2018

Review ended 02/09/2018

Published 02/14/2018

\section{C) Copyright 2018}

Kumar et al. This is an open access article distributed under the terms of the Creative Commons Attribution License CC-BY 3.0., which permits unrestricted use, distribution, and reproduction in any medium, provided the original author and source are credited.

\title{
The Clinical Evaluation of Alcohol Intoxication Is Inaccurate in Trauma Patients
}

Ashwini Kumar ${ }^{1}$, Travis Holloway ${ }^{1}$, Stephen M. Cohn ${ }^{2}$, Gregory Goodwiler ${ }^{1}$, John R. Admire 1

1. Surgery, University of Texas Health Science Center at San Antonio 2. Surgery, Staten Island University Hospital

$\square$ Corresponding author: Stephen M. Cohn, scohn1@northwell.edu

Disclosures can be found in Additional Information at the end of the article

\section{Abstract}

Background: Discharging patients from emergency centers based on the clinical features of intoxication alone may be dangerous, as these may poorly correlate with ethanol measurements.

Objective: We determined the feasibility of utilizing a hand-held breath alcohol analyzer to aid in the disposition of intoxicated trauma patients by comparing serial breathalyzer (Intoximeter, Alco-Sensor FST, St. Louis, Missouri, USA] data with clinical assessments in determining the readiness of trauma patients for discharge.

Methods: A total of 20 legally intoxicated (LI) patients (blood alcohol concentration (BAC) >80 $\mathrm{mg} / \mathrm{dL}$ ) brought to our trauma center were prospectively investigated. Serial breath samples were obtained using a breathalyzer as a surrogate measure of repeated BAC. A clinical exam (nystagmus, one-leg balance, heel-toe walk) was performed prior to each breath sampling.

Results: The enrollees were $85 \%$ male, age $30 \pm 10$ (range 19-51), with a body mass index (BMI) of $29 \pm 7$. The average initial body alcohol level (BAL) was $245 \pm 61$ (range 162-370) $\mathrm{mg} / \mathrm{dL}$. Based on breath samples, the alcohol elimination rates varied from $21.5 \mathrm{mg} / \mathrm{dL} / \mathrm{hr}$ to $45.7 \mathrm{mg} / \mathrm{dL} / \mathrm{hr}$ (mean $28.5 \mathrm{mg} / \mathrm{dL} / \mathrm{hr}$ ). There were no significant differences in alcohol elimination rates by gender, age, or BMI. The clinical exam also varied widely among patients; only seven of 16 (44\%) LI patients demonstrated horizontal nystagmus (suggesting sobriety when actually LI) and the majority of the LI patients (66\%) were able to complete the balance tasks (suggesting sobriety).

Conclusion: Intoxicated trauma patients have an unreliable clinical sobriety exam and a wide range of alcohol elimination rates. The portable alcohol breath analyzer represents a potential option to easily and inexpensively establish legal sobriety in this population.

Categories: Emergency Medicine, General Surgery

Keywords: alcohol, alcohol metabolism, breathalyzer, elimination of ethanol, intoxication, trauma

\section{Introduction}

A high percentage of adults presenting to level I trauma centers are under the influence of alcohol (as high as $50 \%$ at one center (unpublished data). The optimal method to determine 
sobriety and readiness for discharge in patients is uncertain. Some institutions utilize a nomogram based upon a presumed clearance rate to estimate when the patient's serum alcohol level will decrease to the legal limit. Unfortunately, the alcohol metabolism rate varies significantly between individuals, ranging from 13 to $25 \mathrm{mg} / \mathrm{dL} / \mathrm{h}$ in nonalcoholics and 30 to 50 $\mathrm{mg} / \mathrm{dL} / \mathrm{h}$ in alcoholics [1-9]. Other centers rely on a clinical assessment to determine sobriety. After the patient is judged sober, a tertiary exam is done and the patient is discharged home. The clinical features of intoxication may poorly correlate with the ethanol level [9] and, therefore, discharging patients from the ER based on clinical features can be dangerous. Thus, the optimum time for discharging intoxicated patients from the ER has been a subject of debate. Keeping these patients in the ER for prolonged periods comes at the expense of the hospital provider's time, hospital resources, and bed occupancy. On the other hand, the premature discharge of inebriated patients can result in liability for the institution when patients cause harm to themselves or others [10]. All this confusion regarding attaining sobriety in the intoxicated trauma patient creates an undue burden in our already overcrowded Emergency Centers.

We sought to compare serial breathalyzer (Intoximeter, Alco-Sensor FST, St. Louis, Missouri USA) data (sobriety defined as a blood alcohol concentration (BAC) $<80 \mathrm{mg} / \mathrm{dL}$ ) with a clinical assessment in determining the readiness of trauma patients for discharge. Our aim was to determine the feasibility of utilizing a hand-held breath alcohol analyzer to aid in the disposition of intoxicated trauma patients.

\section{Materials And Methods}

Our study protocol was developed at University Hospital, a level I trauma center in San Antonio, Texas, and was approved by the institutional review board (IRB) at the University of Texas Health Science Center. A total of 20 legally intoxicated trauma patients were prospectively enrolled. The patients were approached if they had sustained minimal injury based upon the initial clinical exam and imaging and were unlikely to require admission to the hospital. These patients remained in the emergency department for the entirety of their study involvement. This study did not represent a change in practice; therefore, some of the patients were ultimately admitted for injuries discovered on the tertiary exam or while waiting to become legally sober. An initial determination of legal intoxication was made with the measurement of the blood alcohol concentration (BAC) upon arrival. This is the current standard of care in the trauma population at this emergency department when there is suspicion of intoxication. A BAC greater than $80 \mathrm{mg} / \mathrm{dL}(0.08 \mathrm{~g} / \mathrm{dL})$ was considered legally intoxicated (LI).

\section{Breathalyzer testing}

After the baseline BAC was ascertained, serial breath analyzer testing was performed using an Intoximeter Alco-Sensor FST (cost $=40$ cents/sample). The first breath sample was taken at half the estimated time it takes the individual to eliminate alcohol to the point where the blood alcohol level is below the legal limit (This estimation was based on previously published average clearance rates $(20.43 \mathrm{mg} / \mathrm{dL} / \mathrm{hr})$ ) [8]. Subsequent to the initial breath analysis, serial breath tests were performed at two-hour intervals until a breathalyzer sample was obtained that was below $80 \mathrm{mg} / \mathrm{dL}$.

\section{Clinical testing}

Prior to each breath sample obtained, a three-part clinical exam was administered to assess clinical sobriety. Horizontal nystagmus was tested by placing a finger approximately one foot in front of the patient and moving the finger slowly but steadily horizontally across the patient's field of vision. The examiner instructed the patient to follow the movement with their 
eyes and recorded a positive horizontal nystagmus test if one of the following was met: lack of smooth tracking of the finger, gross nystagmus at the maximal lateral position of the patient's gaze, or the manifestation of nystagmus prior to 45 degrees. Second, a heel-to-toe walking test was performed where patients were instructed to walk the length of their exam room, approximately 15 feet on a line. A positive heel-to-toe walk test was recorded if the patient was unable to walk the complete distance of the line, the patient missed the line during their progression, or there were extreme shifts in balance. Finally, a one-leg balance test was performed where patients were asked to stand on one leg for 10 seconds and repeated using the other leg for an additional 10 seconds. A positive test was recorded if the patient was unable to maintain balance on one leg for the entire preset time or if they had to steady themselves to prevent falling.

A determination of LI was made based on breath analysis results. Once a subject was below the legal limit of alcohol on breath analysis, the clinical testing of intoxication and breath analysis was discontinued and the patient was deemed to have completed the study. All patients were approached again following the conclusion of the study, to ensure there were no questions or changes in consent in the study.

\section{Data analysis}

All patients that required trauma activation were screened for enrollment during the time when research personnel were present. The potential subjects were approached after the conclusion of the primary survey and any initial imaging. The clinical team gave their initial assessment to the research team as to the injuries sustained, initial body alcohol level (BAL), and the likelihood of admission for the injuries sustained. A clinical data sheet was created for each subject. The completion of the clinical examination was recorded as "positive," "negative," or "unable to complete." A breath analysis sample was obtained at the same time as the clinical exam and recorded in $\mathrm{mg} / \mathrm{dL}$.

Using the breath analysis values, a linear slope or best-fit line was determined for each individual (dashed lines) and it was compared to the conservative elimination rate of 20 $\mathrm{mg} / \mathrm{dL} / \mathrm{hr}$ (solid lines). The slope of this line represented the average clearance rate (Figure 1). In addition, the likely time from initial BAL until ethanol clearance was calculated using the previously published $20 \mathrm{mg} / \mathrm{dL} / \mathrm{hr}$ [8]. This time was compared to the time it took for a breath value to reach a level below $80 \mathrm{mg} / \mathrm{dL}$. In addition, subjects were divided by age, gender, and BMI to determine any association with clearance rates. 


\section{Cureus}

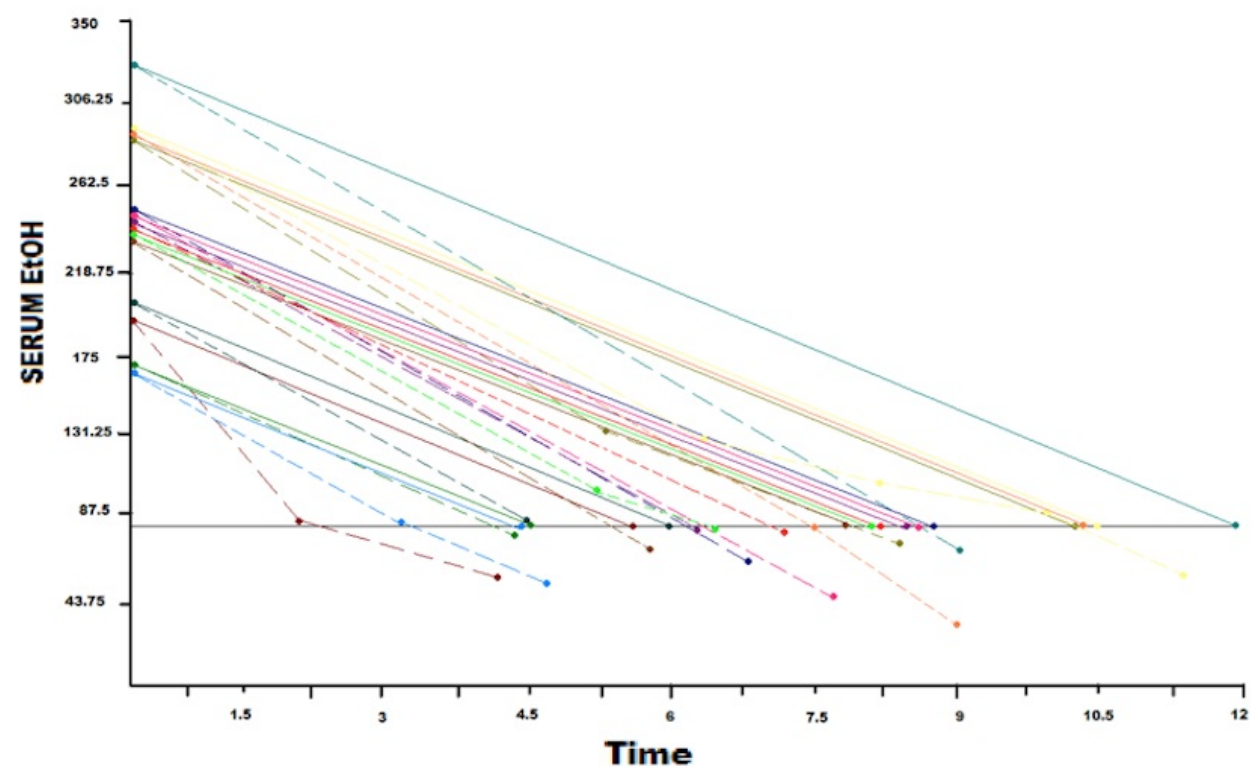

FIGURE 1: Dashed lines represent breath sample measurements, solid lines represent a standard elimination rate of $20 \mathrm{mg} / \mathrm{dl} / \mathrm{hr}$

Different colors represent the individuals.

The Clinical exam results were placed in three separate $2 \times 2$ squares based on the breathalyzer results at the time of that exam. These were analyzed for association.

\section{Results}

Eighty-five percent of the enrollees were male. The average age of the subjects was 30 (range 19-51 years). The average BMI was 28.9, with a standard deviation of 7.1. The average initial BAL was $245 \pm 61$ (range 162-370) $\mathrm{mg} / \mathrm{dL}$. Based on breath samples, the alcohol elimination rates varied from $21.5 \mathrm{mg} / \mathrm{dL} / \mathrm{hr}$ to $45.7 \mathrm{mg} / \mathrm{dL} / \mathrm{hr}$ (average $28.5 \mathrm{mg} / \mathrm{dL} / \mathrm{hr}$ ). When examining the patients in groups based on gender, age, and BMI, there were no statistically significant differences in the elimination rates of alcohol.

The clinical exam varied widely among patients. There was a lack of correlation between breath ethanol (EtOH), nystagmus, balance, and heel-toe tests (Table 1). Of the 16 patients who were able to participate in the horizontal nystagmus test, only seven (44\%) demonstrated a positive horizontal nystagmus. This suggests clinical sobriety; however, these patients continued to have elevated breath alcohol levels (> $80 \mathrm{mg} / \mathrm{dL}$ ). A total of $66 \%$ of the patients who were able to complete the balance tests passed them, despite having breath alcohol levels $>80 \mathrm{mg} / \mathrm{dL}$. Also, of the five patients able to participate, four intoxicated patients (80\%) were able to successfully perform the heel-toe test. This suggests clinical sobriety when these patients continued to have elevated breath alcohol levels and, therefore, none of the clinical exams are sensitive or specific for determining breath EtOH levels and degree of intoxication (Table 1). 


\section{Cureus}

\begin{tabular}{|l|l|l|}
\hline Clinical exam & EtOH level $>80 \mathrm{mg} / \mathrm{dl}$ & EtOH level $<80 \mathrm{mg} / \mathrm{dl}$ \\
\hline Nystagmus (+) & 7 & 9 \\
\hline Nystagmus (-) & 6 & 2 \\
\hline Unable to complete exam & 7 & 20 \\
\hline Total & 20 & EtOH level $<80 \mathrm{mg} / \mathrm{dl}$ \\
\hline Clinical exam & EtOH level $>80 \mathrm{mg} / \mathrm{dl}$ & 9 \\
\hline One leg balance (+) & 4 & 6 \\
\hline One leg balance (-) & 3 & 5 \\
\hline Unable to complete exam & 13 & 20 \\
\hline Total & 20 & EtOH level $<80 \mathrm{mg} / \mathrm{dl}$ \\
\hline Clinical exam & EtOH level $>80 \mathrm{mg} / \mathrm{dl}$ & 8 \\
\hline Heel-toe walk (+) & 4 & 2 \\
\hline Heel-toe walk (-) & 1 & 10 \\
\hline Unable to complete exam & 15 & 20 \\
\hline Total & 20 & \\
\hline
\end{tabular}

TABLE 1: The three clinical exams conducted were for the presence of nystagmus, ability to balance on one leg, and ability to walk heel to toe. The ability to conduct each exam did improve when patients reached legal sobriety (EtOH $<80 \mathrm{mg} / \mathrm{dL}$ ); however, none of the tests were sensitive or specific for intoxication in our patient population.

EtOH: breath ethanol

\section{Discussion}

Alcohol ingestion directly affects risk-taking behavior in humans [11]. It has been proven that there is a time-course effect of alcohol dose on attention span, impulsivity, discrimination, and response time [12]. The optimum time for discharging intoxicated patients from the ER remains a subject of considerable debate. In our study, we found that alcohol elimination rates varied widely (21.5 mg/dL/hr to $45.7 \mathrm{mg} / \mathrm{dL} / \mathrm{hr}$ ) among our trauma patients and that clinical examination was completely unreliable in predicting blood alcohol concentration.

Enzymatic oxidation in the liver is the principal path of elimination of alcohol; a small quantity of unmetabolized alcohol is excreted in the urine, breath, and perspiration [13]; which permits alcohol concentration to be measured in breath and urine. Quantitative data on alcohol elimination has been obtained, mostly from the repeated measurement of blood or breath 
alcohol concentrations, but a range of clearance rates have been reported $[9,12]$.

Currently, the criteria for discharging intoxicated patients vary between institutions. While some rely on clinical evaluation for this purpose, others wait for the alcohol level to fall under legal limits. Clinical suspicion begins with the measurement of BAC during the initial exam and if injuries are minor, alcohol concentration, along with clinical examinations, are consecutively performed until they reach safe levels prior to discharge. Unfortunately, the results of clinical examination appear poorly correlated with the actual intoxication of the patient [9] and without adjuvant objective data, can overestimate the level of sobriety in this population. Discharging patients from the ER based on clinical judgment alone can also lead to errors in management, placing patients at further risk of injury.

We are often faced with difficulties relating to patient overflow in our emergency centers and trouble with the disposition of patients after minor trauma. This raises the question of how to properly manage patients who are legally intoxicated and awaiting sobriety in our ED and trauma departments. With the wide range of clearance rates and no universal consensus, some patients are receiving multiple blood draws, which increases the cost of care and the unnecessary utilization of resources. The time factor is also considerable during the clearance in these patients; while awaiting definitive management, some patients are inappropriately admitted in order to free up space in the ED.

To mitigate risk, cost, and time management, we have found that a breathalyzer is both clinical and cost-effective. Despite the relatively high initial cost (USD 555), it can be used repeatedly and reduce the cost of care without affecting quality. It can be utilized in the ED with very little training required, and the results are immediate. Breathalyzers are commonly used in the field by law enforcement but can be utilized in the hospital setting as an ancillary diagnostic tool along with clinical evaluation.

\section{Limitations}

Although the breathalyzer is easy to operate, appropriate training needs to be provided to emergency room staff. The breathalyzer should be calibrated every six months to ensure appropriate functioning; otherwise, the machine may give faulty readings. Also, alcohol absorption may vary from a few minutes to over two hours after consumption, depending on the type and amount of food ingested. These factors should be taken into account while performing the breathalyzer test.

\section{Conclusions}

Given the wide range of alcohol elimination rates and the inaccuracy of clinical exams, a portable alcohol breath analyzer has the potential to establish legal sobriety in the trauma population. This instrument is already being used by law enforcement, is readily available, easy to use, and inexpensive.

\section{Appendices}

1. Why is this topic important?

The results of clinical examinations are poorly correlated with the actual intoxication of the patient, and some patients receive multiple blood draws, which only amounts to an increased cost of care and the unnecessary utilization of resources. Discharging patients from the ER based on clinical judgment alone can also lead to errors in management, placing patients at further risk of injury. 
2. What does this study attempt to show?

The breathalyzer is both clinical and cost-effective. It can be utilized in the ED with very little training. Breathalyzers are commonly used in the field by law enforcement but can be utilized in the hospital setting as an ancillary diagnostic tool along with clinical evaluation.

3. What are the key findings?

There were no statistically significant differences in the elimination rates of alcohol when examining patients in groups based on gender, age, and BMI. None of the subjects metabolized EtOH slower than $20 \mathrm{mg} / \mathrm{dL} / \mathrm{hr}$. Also, the clinical exam varied widely among patients. In addition, a breathalyzer is both clinical and cost-effective.

\section{How is patient care impacted?}

The use of breathalyzers can help determine the appropriateness of discharge timing for inebriated patients and can reduce the cost of care, without affecting quality.

\section{Additional Information Disclosures}

Human subjects: Consent was obtained by all participants in this study. Institutional Review Board of University of Texas Health Science Center, San Antonio issued approval HSC20110163H. Dear Principal Investigator, Your request to conduct this minimal risk research was approved by Expedited Review on January 13, 2011, under the following regulation(s): 45 CFR 46.110(b)(1) Category 4: Collection of data through non invasive procedures routinely employed in clinical practice, excluding procedures involving x-rays or microwaves. 45 CFR 46.110(b)(1) Category 5: Research involving materials (data, documents, records or specimens) that have been collected, or will be collected solely for non-research purposes (such as medical treatment or diagnosis). A waiver of requirement to obtain a valid authorization to use or disclose PHI was also approved. The IRB has determined that the following criteria as required by 45CFR164.512(i) were satisfied: $\mathbf{X}$-The PHI use or disclosure involves no more than minimal risk to the privacy of individuals based on at least the presence of (1) an adequate plan presented to the Privacy Board to protect PHI identifiers from improper use and disclosure; (2) an adequate plan to destroy those identifiers at the earliest opportunity, consistent with the research, absent a health or research justification for retaining the identifiers or if retention is otherwise required by law; and (3) adequate written assurances that the PHI will not be reused or disclosed to any other person or entity except (a) as required by law, (b) for authorized oversight of the research study, or (c) for other research for which the use or disclosure of the PHI is permitted by the Privacy Rule. $\mathbf{X}$-The research could not practicably be conducted without the requested waiver or alteration. $\mathbf{X}$-The research could not practicably be conducted without access to and use of the PHI. A waiver of documentation of consent is approved based on meeting the requirements specified by 45 CFR 46.117 (c). Animal subjects: All authors have confirmed that this study did not involve animal subjects or tissue. Conflicts of interest: In compliance with the ICMJE uniform disclosure form, all authors declare the following:

Payment/services info: All authors have declared that no financial support was received from any organization for the submitted work. Financial relationships: All authors have declared that they have no financial relationships at present or within the previous three years with any organizations that might have an interest in the submitted work. Other relationships: All authors have declared that there are no other relationships or activities that could appear to have influenced the submitted work. 


\section{References}

1. Holtzman JL, Gebhard RL, Eckfeldt JH, Mottonen LR, Finley DK, Eshelman FN: The effects of several weeks of ethanol consumption on ethanol kinetics in normal men and women. Clin Pharmacol Ther. 1985, 38:157-163. 10.1038/clpt.1985.152

2. Winek CL, Murphy KL: The rate and kinetic order of ethanol elimination . Forensic Sci Int. 1984, 25:159-166. 10.1016/0379-0738(84)90189-0

3. Olsen H, Sakshaug J, Duckert F, Stromme JH, Morland J: Ethanol elimination-rates determined by breath analysis as a marker of recent excessive ethanol consumption. Scand J Clin Lab Invest. 1989, 49:359-365. 10.3109/00365518909089109

4. Rangno RE, Kreeft JH, Sitar DS: Ethanol 'dose-dependent' elimination: Michaelis-Menten v classical kinetic analysis. Br J Clin Pharmacol. 1981, 12:667-673. 10.1111/j.1365-

2125.1981.tb01287.x

5. Kater RM, Carulli N, Iber FL: Differences in the rate of ethanol metabolism in recently drinking alcoholic and nondrinking subjects. Am J Clin Nutr. 1969, 22:1608-1617.

6. Cole-Harding S, Wilson JR: Ethanol metabolism in men and women . J Stud Alcohol. 1987, 48(4):380-7. 10.15288/jsa.1987.48.380

7. Dubowski KM: Absorption, distribution and elimination of alcohol: highway safety aspects . J Stud Alcohol Suppl. 1985, 10:98-108. 10.15288/jsas.1985.s10.98

8. Gershman H, Steeper J: Rate of clearance of ethanol from the blood of intoxicated patients in the emergency department. J Emerg Med. 1991, 9:307-311. 10.1016/0736-4679(91)90371-L

9. Brennan DF, Betzelos S, Reed R, Falk JL: Ethanol elimination rates in an ED population. Am J Emerg Med. 1995, 13:276-280. 10.1016/0735-6757(95)90199-X

10. Marco CA, Kelen GD: Acute intoxication. Emerg Med Clin North Am. 1990, 8:731-748.

11. Lane SD, Cherek DR, Pietras CJ, Tcheremissine OV: Alcohol effects on human risk taking . Psychopharmacology (Berl). 2004, 172:68-77.

12. Dougherty DM, Marsh DM, Moeller FG, Chokshi RV, Rosen VC: Effects of moderate and high doses of alcohol on attention, impulsivity, discriminability, and response bias in immediate and delayed memory task performance. Alcohol Clin Exp Res. 2000, 24:1702-1711. 10.1111/j.1530-0277.2000.tb01972.x

13. Hawkins RD, Kalant H: The metabolism of ethanol and its metabolic effects . Pharmacol Rev. 1972, 24:67-157. 